\title{
Dirty-paper Coding Techniques for Compound MISO Broadcast Channels: A DoF Analysis
}

\author{
Meryem Benammar Pablo Piantanida \\ Dept. of Telecommunications, SUPELEC \\ 91192 Gif-sur-Yvette, France \\ Email:\{meryem.benammar, pablo.piantanida\}@supelec.fr
}

\author{
Shlomo Shamai (Shitz) \\ Department of Electrical Engineering \\ Technion, Haifa, Israel \\ Email:sshlomo@ee.technion.ac.il
}

\begin{abstract}
This work investigates optimal Degrees of Freedom (DoF) achieving schemes for the MISO Compound Broadcast Channels (BC) where a source is equipped with $M$ antennas, and communicates with 2 single antennas receivers. We develop a modified Dirty Paper Coding scheme that yields the optimal DoF for some Compound MISO BCs in the complex field. This coding scheme offers a "non-linear" alternative to interference alignment at the receivers which yields higher rates in the finite ranges of power. For asymptotic power regimes, it allows for fractional DoF of $1 / 2$, while being of simple formulation and straightforward application to secondary transmissions in Compound Cognitive networks.
\end{abstract}

\section{INTRODUCTION}

We consider in this work the MISO Broadcast Channel where a source equipped with $M$ antennas transmits dedicated messages to two single antenna receivers. The channels' realizations at each of the receivers depend on a finite state, of which the source has no prior knowledge (no CSIT), and supposed to remain constant throughout the transmission. Communicating successfully over this network imposes that whatever the realizations of the channels - the users be able to decode perfectly the messages, leading to a "worst case" performance known as the "Compound" capacity region.

Applications of this setting arise in most recent cellular networks, where a multiple antennas source transmits both common and dedicated packets to single antenna terminals while being totally unaware of the link "quality" available at each user. Besides, in such Broadcast settings, the transmitter deals with interfering messages in the same way a Cognitive Secondary source - having access to the message of the Primary source as side information - transmits to the Secondary Users with little impediment to the Primary User's rate.

The MIMO Broadcast Channel capacity region is due to Weingarten et al. [1], however, the Compound MIMO BC capacity region remains unknown to this day for a general setting, though, it was derived for a few degraded cases where a specific order is imposed on the channels of the two users [2], [3]. Relating to our setting, the Compound MISO BC, when not trivial, i.e. when the channel instances of each users are not collinear, does not fall in any of these two classes and thus little is yet known about its capacity region. However,

The work of P. Piantanida and S. Shamai was partially supported by the FP7 Network of Excellence in Wireless COMmunications NEWCOM\#. the Degrees of Freedom (DoF) analysis can be insightful to understand how interference should be managed with no CSIT.

The effect of channel uncertainty on the DoF is rather well understood. For finite state Compound settings, Weingarten et al. had first derived both inner and outer bounds on the DoF region and on the sum-DoF of the Compound MISO BC [4] with some cases of optimality. The outer bound derived therein was conjectured to be loose, but later Maddah-Ali [5] proved the optimal DoF region of the generic Compound MISO BC, both in the complex and in the real settings, to perfectly match this outer bound. The achievability of the optimal DoF relies on either Linear or non-Linear coding scheme combined with Symbol Extensions in [4] while the proof made in [5] resorts to Number Theory results and consists in interference alignment over rational dimensions of the real numbers, see also [6]. When the states span an infinite set, i.e. in the ergodic setting, DoF can experience severe loss, and in [7], it is shown that with Rayleigh fading channels, the sum-DoF collapses to the number of transmit antennas: Time Sharing is optimal. A few more works deal with various models of the amount and accuracy of CSI available at the transmitter, e.g. [8].

In this work, we aim at characterizing the role that "nonlinear" coding, mainly based on "writing on dirty paper" [9] or its vector formulation " writing on coloured paper" [10] and that we call throughout this work Dirty Paper Coding (DPC), can play in achieving optimal DoF in the complex Compound MISO BC. We can infer that "non-linear" coding schemes will outperform linear coding schemes, as they do clearly for the standard MIMO BC [1] when considering finite ranges of transmitting power. The mere application of DPC on the Compound setting leads in general to integer valued DoF 0 or 1 , unless combined with a time sharing argument. In this work we show that, upon modifying the code construction argument in DPC, we can obtain fractional DoF of $1 / 2$ as well as 1 or 0 DoF. This allows us to recover the optimal DoF for the general 3 by 1 and a class of 4 by 1 Compound MISO BC.

The remainder of the paper is organised as follows. Definitions and previous results on DoF are given in Section II. In Section III, an inner bound on the capacity region of the Compound MISO BC is derived. We then analyse the resulting DoF and compare the suggested scheme to Interference Alignment in Section IV. Last, conclusions are drawn in Section V. 


\section{Notations}

In the sequel, (RV) resp. (ARV) stands for Random variable resp. Auxiliary Random Variable. $\mathbf{h}^{t}$ is to be understood as the transpose of the vector $\mathbf{h}$, while $X$ denotes a scalar random variable. $\mathbf{X}^{H}$ reads as the Hermitian of the random vector $\mathbf{X}$, plus, the scalar product of the vectors $\mathbf{h}$ and $\mathbf{B}_{u}$ writes as : $\mathbf{h}^{t} \mathbf{B}_{u}=h_{u}$. The notation $|\alpha|$ stands for the absolute value of the complex number $\alpha$ and $\alpha^{*}$ for its conjugate when $\Im(\alpha)$ and $\Re(\alpha)$ designate its imaginary and real part. Last, the set $\{1, \ldots, J\}$ denotes the set of integers ranging from 1 to $J$.

\section{SYSTEM MODEL}

\section{A. $J_{y}$ by $J_{z}$ Compound Broadcast Channel}

Let us consider the following Compound MISO Broadcast Channel consisting of a source equipped with $M$ antennas and 2 single antenna receivers $Y$ and $Z$ each having uncertainty about their actual channel. Denote the respective finite numbers of possible channels at users as $J_{y}$ and $J_{z}$.

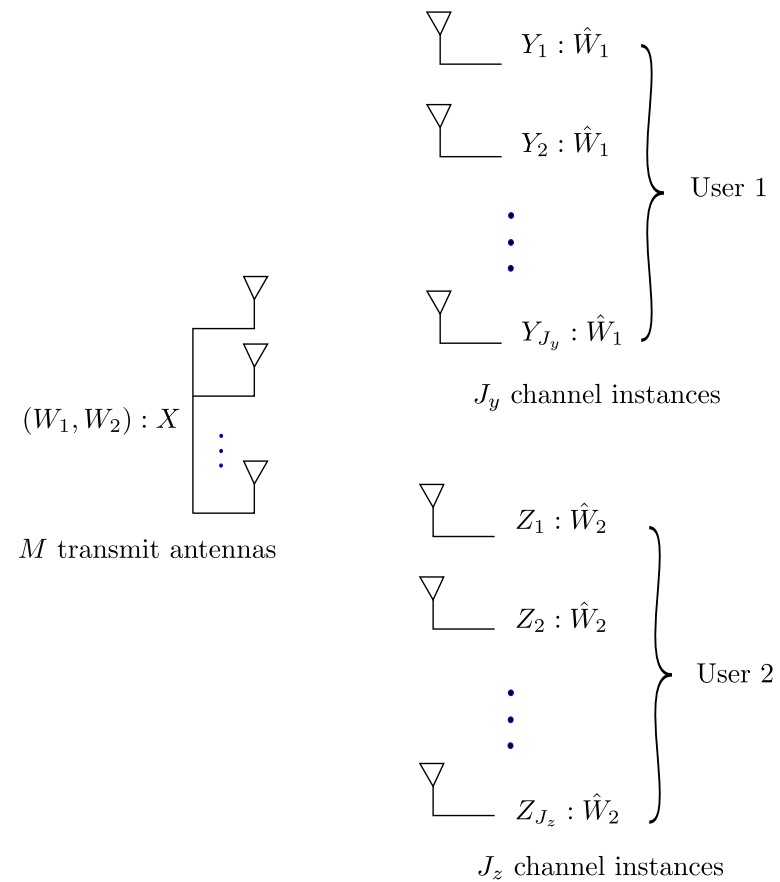

Fig. 1. The $J_{y}$ by $J_{z}$ Compound MISO Broadcast Channel

The channel outputs at time $n$ are given by:

$$
\begin{array}{ll}
\forall j \in\left\{1, \ldots, J_{y}\right\} & y_{j, n}=\mathbf{h}_{j}^{t} \mathbf{x}_{n}+n_{j, n}, \\
\forall k \in\left\{1, \ldots, J_{z}\right\} & z_{k, n}=\mathbf{g}_{k}^{t} \mathbf{x}_{n}+w_{k, n},
\end{array}
$$

where:

- $\mathbf{h}_{j}$ and $\mathbf{g}_{k}$ are $2 \times 1$ generic complex valued channel vectors, i.e. any subset of $M$ channels among them are linearly independent. They are supposed constant throughout the transmission.

- $\mathbf{X}$ is a $2 \times 1$ complex valued channel input vector. We suppose that the input is power constrained so that $\mathbb{E}\left(\mathbf{X}^{H} \mathbf{X}\right) \leq P$

- The model being rich enough with the generic channel coefficients, the noise sequences $N_{j}^{(n)}$ and $W_{k}^{(n)}$ are supposed
TABLE I

SUM-DoF OF THE COMPOUND MISO BC $\left(J_{y}, J_{z}\right)$

\begin{tabular}{|c|c|c|}
\cline { 2 - 3 } \multicolumn{1}{c|}{} & $J_{y}<M$ & $J_{y} \geq M$ \\
\hline$J_{z}<M$ & 2 & $1+\frac{M-1}{M}$ \\
\hline$J_{z} \geq M$ & $1+\frac{M-1}{M}$ & $\frac{2 M}{M+1}$ \\
\hline
\end{tabular}

to be i.i.d draws of a white circular symmetric Gaussian process $\mathcal{C N}(0, N)$.

As pointed out previously, we assume the receivers to have perfect knowledge of their respective channels (full CSIR). Since no form of CSIT is considered, i.e the source is totally oblivious to the channels controlling the communication, this setting becomes then equivalent, in terms of maximum error probability, to a Broadcast Channel with common messages where all users $Y_{j}$ are interested in message $W_{1}$ and all $Z_{k}$ are interested in $W_{2}$. Fig 1 plots the channel model.

We will denote such a system by the couple $\left(J_{y}, J_{z}\right)$ and call it the $J_{y}$ by $J_{z}$ Compound MISO BC, where it is well understood that the number of antennas at the source is $M$.

\section{B. DoF Region and sum-DoF}

We can define both a DoF Region and a sum-DoF copying the behaviour of the capacity region and of the sum capacity at very high powers. In the complex setting, the DoF regions is the convex closure of the set given by:

$\mathcal{D} \triangleq\left\{\lim _{P \rightarrow \infty}\left(\frac{R_{1}(P)}{\log _{2}(P)}, \frac{R_{2}(P)}{\log _{2}(P)}\right),\left(R_{1}(P), R_{2}(P)\right) \in \mathcal{C}(P)\right\}$

The sum-DoF can be defined in a similar fashion:

$D \triangleq \max \left\{\lim _{P \rightarrow \infty} \frac{R_{1}(P)+R_{2}(P)}{\log _{2}(P)},\left(R_{1}(P), R_{2}(P)\right) \in \mathcal{C}(P)\right\}$

DoF is a rather accommodating performance measure since it infers on the number of interference-free links available at the source to convey the messages to their respective users.

The optimal DoF region for the complex MISO BC depends on the number of instances at each user and of how they relate to the number of antennas at the transmitter.

For the cases where $J_{y}=1, J_{z}=M$ and $J_{y}=M, J_{z}=M$, the optimal DoF regions are due to [4], whereas for more general number of instances at each users, the optimal sumDoF are due to [5]. These results are summarized in Table. I.

It can be noticed here that the sum-DoF is not much affected by the number of instances at each user and that it remains always greater than 1 for $M \geq 2$.

In the sequel, we explore another way of achieving the optimal DoF in the case where only one user has uncertainty about his channel, say $J_{z}=1$, and where the source has $M=2$ antennas. The optimal sum-DoF is then equal to $3 / 2$, which equivalently consists in the DoF region corner point $(1 / 2,1)$ as shown in Fig 2. 


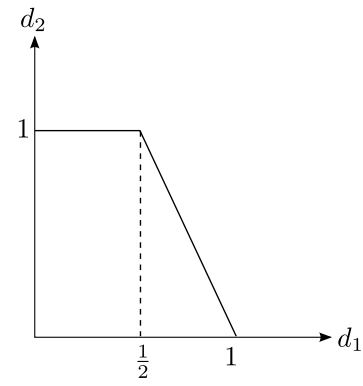

Fig. 2. The DoF region of the 2 by 1 Compound MISO Broadcast Channe

\section{INNER BOUND ON THE CAPACITY REGION OF THE COMPOUND MISO BROADCAST CHANNEL}

In this section we derive an inner bound based on a coding scheme that combines both DPC and linear precoding.

Theorem 1. An inner bound on the capacity region of the Compound Gaussian MISO broadcast channel consists of all rate pairs satisfying:

$$
\begin{aligned}
R_{1} & \leq \frac{1}{2} \max _{(\alpha, \beta) \in \mathbb{C}^{2}} \min _{j \in\left\{1, \ldots, J_{y}\right\}} \log _{2}\left(\frac{P_{u}^{2}}{f_{j}^{-}(\alpha, \beta) f_{j}^{+}(\alpha, \beta)}\right) \\
R_{2} & \leq \log _{2}\left(\frac{\left|g_{u}\right|^{2} P_{u}+\left|g_{v}\right|^{2} P_{v}+N}{\left|g_{u}\right|^{2} P_{u}+N}\right)
\end{aligned}
$$

where :

$$
\begin{gathered}
f_{j}^{-}(\alpha, \beta)=\frac{P_{v}\left(\left|h_{j, u}\right|^{2} P_{u}+N\right)}{\left|h_{j, u}\right|^{2} P_{u}+\left|h_{j, v}\right|^{2} P_{v}+N}\left(\left|\alpha-\alpha_{j}\right|-|\beta|\right)^{2} \\
+\frac{N P_{u}}{\left|h_{j, u}\right|^{2} P_{u}+N} \\
f_{j}^{+}(\alpha, \beta)=\frac{P_{v}\left(\left|h_{j, u}\right|^{2} P_{u}+N\right)}{\left|h_{j, u}\right|^{2} P_{u}+\left|h_{j, v}\right|^{2} P_{v}+N}\left(\left|\alpha-\alpha_{j}\right|+|\beta|\right)^{2} \\
+\frac{N P_{u}}{\left|h_{j, u}\right|^{2} P_{u}+N}
\end{gathered}
$$

and, $\alpha_{j}=\frac{P_{u} h_{j, u}^{*} h_{j, v}}{\left|h_{j, u}\right|^{2} P_{u}+N_{y}}$ and $P_{u}+P_{v} \leq P$.

Proof. We give here the main elements of proof, the remainder of which is purely technical and is presented in Appendix. A.

Inspired by Marton's inner bound for the general broadcast channel [11], we can derive a corner point of what we call the "worst case" Marton's inner bound that consists of all rate pairs $\left(R_{1}, R_{2}\right)$ satisfying:

$$
\left\{\begin{array}{l}
R_{1} \leq \min _{j \in\left\{1, . ., J_{y}\right\}} I\left(U ; Y_{j} \mid Q\right)-I(U ; V \mid Q) \\
R_{2} \leq I(V ; Z \mid Q)
\end{array}\right.
$$

for some joint p.m.f factoring as: $p_{Q U V X}=p_{Q U V} \cdot p_{X \mid Q U V}$.

To achieve the rate region (2), one has to evaluate the previous region using the following coding scheme:

$$
\left\{\begin{aligned}
\mathbf{X} & =\mathbf{X}_{u}+\mathbf{X}_{v} \\
\mathbf{X}_{u} & =X_{u} \mathbf{B}_{u} \\
\mathbf{X}_{v} & =X_{v} \mathbf{B}_{v} \\
U & =X_{u}+\alpha X_{v}+\underline{\beta X_{v}^{*}} \\
V & =X_{v}
\end{aligned}\right.
$$

where $X_{u} \sim \mathcal{C N}\left(0, P_{u}\right)$ and $X_{v} \sim \mathcal{C N}\left(0, P_{v}\right)$ such that $P_{u}+$ $P_{v} \leq P$. Plus, $\mathbf{B}_{u}$ and $\mathbf{B}_{v}$ are unit norm complex beams, and the common $\operatorname{ARV} Q$ is set to a trivial value.

The main difference with the vector DPC - a part from the unit rank covariance matrices - is that we allow to compensate a part of interference and of its conjugate, the reason of which will be justified in the DoF analysis.

Unlike in the scalar DPC where the optimal parameter $\alpha$ does not depend on the interference, the optimal vector DPC parameter depends on the interference in the way it affects the channel $\mathbf{h}_{j}$. In other terms, if $\mathbf{h}_{j}$ and $\mathbf{B}_{v}$ were to be orthogonal, there would be no need for cancelling the interference since it does not affect the channel output.

In the "non-Compound" setting, i.e $J_{y}=1$, the maximizing choice of the parameters is $\alpha=\alpha_{1}$ and $\beta=0$, which copies the standard DPC for the MISO setting. Note here though, that in that case, we are evaluating the capacity region of the MISO broadcast with unit rank covariance matrices, which, except for the individual rates and the sum rate [12], does not guarantee optimality. Yet, the result based on Beamforming is strong enough to yield the optimal DoFs as will be discussed.

\section{DOF ANALYSIS OF THE COMPOUND BROADCAST CHANNEL}

In this section we analyse the DoF region obtained from Theorem 1 and discuss the achievability of the corner point $\left(d_{1}=1 / 2, d_{2}=1\right)$ with this result.

It is clear that the DoF of user 2 can be made equal to 1 by letting $g_{u}=0$, i.e by transmitting the signal intended to user 1 in a direction orthogonal to the 2nd user's channel.

For the case of 2 by 1 Compound MISO, it can be shown that a time-sharing argument along with linear precoding (zero-forcing) allows to achieve the optimal $1 / 2$ DoF for user 1 . With the less trivial cases of 3 by 1 and 4 by 1 Compound MISO BC, comes the need for more evolved coding schemes such as interference alignment, or alternately the scheme suggested in the proof of theorem 1 and that we analyse hereafter.

\section{A. Conditions of optimality}

To derive the achievable DoF of user 1 , we need to analyse the DoF loss in each of the two terms: $f_{j}^{+}(\alpha, \beta)$ and $f_{j}^{-}(\alpha, \beta)$ for each instance $\mathbf{h}_{j}$ of the channel, in the presence of scalable interference, i.e. $P_{v}$ scales with $P$. Note that:

- If $|\beta|+\left|\alpha-\alpha_{j}\right| \neq 0$, then $f_{j}^{+}(\alpha, \beta)$ scales with power, and thus leads to $\frac{1}{2}$ DoF loss out of the available 1 DoF.

- Otherwise, if $|\beta|+\left|\alpha-\alpha_{j}\right|=0$, then this term results in no loss of DoF. The same reasoning holds for the term $f_{j}^{-}(\alpha, \beta)$.

In Table II lies a summary of the DoF loss in each term of the rate of user 1 following the respective values of the parameters of the DPC: $\alpha$ and $\beta$.

The achievable DoF with such a setting will then relate directly to the feasiblity of the conditions in table II for the many instances of channels of user 1 .

It can be readily checked that since the beam $\mathbf{B}_{u}$ is already fixed orthogonal to the channel instance $\mathrm{g}$, there is only one 
TABLE II

DoF LOSS IN THE TERMS $f_{j}^{+}(\alpha, \beta)$ AND $f_{j}^{-}(\alpha, \beta)$ AS A PAIR $\left(d^{+}, d^{-}\right)$

\begin{tabular}{|c|c|c|}
\cline { 2 - 3 } \multicolumn{1}{c|}{} & $|\beta|=0$ & $|\beta| \neq 0$ \\
\hline$|\beta|=\left|\alpha-\alpha_{j}\right|$ & $(0,0)$ & $(1 / 2,0)$ \\
\hline$|\beta| \neq\left|\alpha-\alpha_{j}\right|$ & $(1 / 2,1 / 2)$ & $(1 / 2,1 / 2)$ \\
\hline
\end{tabular}

complex dimension left, spanned by the beam $\mathbf{B}_{v}$, to fetch as many constraints as possible. The following lemma enunciates our first statement.

\section{Lemma 1. The following constraint}

$$
|\beta|=\left|\alpha-\alpha_{j}\right|=\left|\alpha-\alpha_{i}\right|=0 \quad \text { for at least some } i \neq j
$$

does not yield the optimal sum-DoF.

Proof. The proof of this statement is two fold.

- First, suppose that there exist a couple of indices $(i, j)$ whose channels are not collinear and that satisfy: $\alpha_{i}=\alpha_{j}$. This means in other terms that:

$$
\begin{aligned}
& \frac{P_{u} h_{i, u}^{*} h_{i, v}}{\left|h_{i, u}\right|^{2} P_{u}+N_{y}}=\frac{P_{u} h_{j, u}^{*} h_{j, v}}{\left|h_{j, u}\right|^{2} P_{u}+N_{y}} \\
& \left(\frac{P_{u} h_{i, u}^{*}}{\left|h_{i, u}\right|^{2} P_{u}+N_{y}} \mathbf{h}_{i}^{t}-\frac{P_{u} h_{j, u}^{*}}{\left|h_{j, u}\right|^{2} P_{u}+N_{y}} \mathbf{h}_{j}^{t}\right) \times \mathbf{B}_{v}=0 .
\end{aligned}
$$

- Now in high SNR regimes, the previous equality becomes equivalent to:

$$
\left(\frac{1}{h_{i, u}} \mathbf{h}_{i}^{t}-\frac{1}{h_{j, u}} \mathbf{h}_{j}^{t}\right) \times \mathbf{B}_{v}=0 .
$$

But since $\mathbf{B}_{u}$ is already a solution to this equation, it imposes that $\mathbf{B}_{v}$ be collinear to $\mathbf{B}_{u}$. Thus $\mathbf{B}_{v}$ is orthogonal to the channel of the second user 2, which leads to a $0 \mathrm{DoF}$ for this user, leading thus to a sum-DoF of 1 in stead of the expected $3 / 2$. Thus, though it might have seemed at first glance that there could be a case where all the $\alpha_{j}$ are equal (by letting $\mathbf{B}_{u}=\mathbf{B}_{v}$ ), which would lead indeed to 1 DoF for all the instances of user 1 but this is strictly sub-optimal from a sumDoF standpoint.

In the sequel, it is well understood then that the parameters $\alpha_{j}$ are all pairwise distinct.

\section{B. The 3 by 1 MISO Compound BC}

Let us assume first, that user 1 can observe one of 3 possible channel outputs. The following corollary shows how to achieve the optimal 3/2 sum-DoF for the Compound MISO BC under study.

Corollary The following choice of parameters

$$
\begin{aligned}
& \alpha=\frac{\left(\alpha_{1}-\alpha_{3}\right)\left(\left|\alpha_{1}\right|^{2}-\left|\alpha_{2}\right|^{2}\right)-\left(\alpha_{1}-\alpha_{2}\right)\left(\left|\alpha_{1}\right|^{2}-\left|\alpha_{3}\right|^{2}\right)}{2 i \Im\left(\left(\alpha_{1}-\alpha_{3}\right)\left(\alpha_{1}-\alpha_{2}\right)^{*}\right)} \\
& |\beta|=\frac{\left|\alpha_{1}-\alpha_{2}\right|\left|\alpha_{2}-\alpha_{3}\right|\left|\alpha_{1}-\alpha_{3}\right|}{2\left|\Im\left(\left(\alpha_{1}-\alpha_{3}\right)\left(\alpha_{1}-\alpha_{2}\right)^{*}\right)\right|}
\end{aligned}
$$

leads along with Theorem 1 to the optimal DoF for the 3 by 1 Compound MISO BC.
Proof. Achieving the optimal DoF of $1 / 2$ relates mainly to the condition: $\forall i \in\{1, . ., 3\},|\beta|=\left|\alpha-\alpha_{j}\right|$. Satisfying such a condition means finding a complex number "equidistant" from the three complex quantities $\alpha_{j}{ }^{1}$. One can easily check that the suggested couple of parameters satisfies this condition, we thus conclude that one can achieve the optimal DoF for the 3 by 1 Compound MISO BC. Note here though that the beam $\mathbf{B}_{v}$ has not been optimized over yet; we only imposed that it be not orthogonal to the channel of the second user $\mathrm{g}$.

With this choice of parameters, we can accommodate all channels $\mathbf{h}_{j}$ such that the following orthogonality holds:

$$
\left(\exists \theta_{j} \in[0: 2 \pi]\right), \mathbf{h}_{j}^{t} \times\left(\mathbf{B}_{v}+\left(\alpha+e^{i \theta_{j}}|\beta|\right) \mathbf{B}_{u}\right)=0 .
$$

Recall the dependence of $\alpha$ and $\beta$ on $\mathbf{B}_{v}$ through the $\alpha_{j}$ s.

\section{Towards the 4 by 1 Compound MISO BC}

The key point for showing the achievability of the $1 / 2 \mathrm{DoF}$ for the 4 by 1 Compound MISO BC, is to show that, for an instance $\mathbf{h}_{4}$, there exists a beam $\mathbf{B}_{v}$ such that (5) holds.

The following lemma gives the class of Compound MISO $\mathrm{BC}$ for which the rate region of Theorem 1 is DoF optimal.

Lemma 2. For any complex vector channel $\mathbf{h}_{4}$, (2) is DoF optimal if and only if (5) holds for $\mathbf{B}_{v}=\mathbf{B}_{u}^{\perp}=\frac{\mathbf{g}}{\|\mathbf{g}\|}$.

Proof. Let us first introduce some notations. Let $\mathbf{B}_{u}^{\perp}$ denote the unit-norm vector orthogonal to $\mathbf{B}_{u}$. Let also $h_{j, u}^{\perp}$ denote the new scalar product $h_{j, u}^{\perp}=\mathbf{h}_{j}^{t} \mathbf{B}_{u}^{\perp}$. Last, we note :

$$
h_{j}=\frac{h_{j, u}^{\perp}}{h_{j, u}}=\frac{\mathbf{h}_{j}^{t} \mathbf{B}_{u}^{\perp}}{\mathbf{h}_{j}^{t} \mathbf{B}_{u}} .
$$

Since $\mathbf{B}_{u}$ and $\mathbf{B}_{u}^{\perp}$ form an orthonormal basis of the space of complex vectors, let us write for each vector $\mathbf{B}_{v}$ :

$$
\mathbf{B}_{v}=u \mathbf{B}_{u}+v \mathbf{B}_{u}^{\perp},
$$

where $|u|^{2}+|v|^{2}=1$. At high power, all the $\alpha_{j}$ write as:

$$
\alpha_{j}=\frac{\mathbf{h}_{j}^{t} \mathbf{B}_{v}}{\mathbf{h}_{j}^{t} \mathbf{B}_{u}}=u+v h_{j} .
$$

Then, one can rewrite the optimal parameters $\alpha$ and $|\beta|$ explicitly in $(u, v)$ as:

$$
\begin{aligned}
& \alpha=u+v h^{\perp} \\
& |\beta|=|v| d^{\perp},
\end{aligned}
$$

where the point $h^{\perp}$ and the distance $d$ are defined as:

$$
\begin{aligned}
h^{\perp} & =\frac{\left(h_{1}-h_{3}\right)\left(\left|h_{1}\right|^{2}-\left|h_{2}\right|^{2}\right)-\left(h_{1}-h_{2}\right)\left(\left|h_{1}\right|^{2}-\left|h_{3}\right|^{2}\right)}{2 i \Im\left(\left(h_{1}-h_{3}\right)\left(h_{1}-h_{2}\right)^{*}\right)} \\
d^{\perp} & =\frac{\left|h_{1}-h_{2}\right|\left|h_{1}-h_{3}\right|\left|h_{2}-h_{3}\right|}{2\left|\Im\left(\left(h_{1}-h_{3}\right)\left(h_{1}-h_{2}\right)^{*}\right)\right|} .
\end{aligned}
$$

Note here that $h^{\perp}$ is nothing but the very optimal parameter $\alpha$ when evaluated with $\mathbf{B}_{v}=\mathbf{B}_{u}^{\perp}$ and so is the distance $d^{\perp}$.

\footnotetext{
${ }^{1}$ Here distance stands for the Euclidean distance, regarding the Complex space as a two-dimensional Real space.
} 
We can proceed now with the proof of our claim. Suppose (5) holds; it can be written as:

$$
\exists\left(\mathbf{B}_{v}, \theta_{4}\right) \quad \alpha_{4}=\alpha+e^{i \theta_{4}}|\beta| .
$$

which means that for some $(u, v)$ :

$$
\begin{aligned}
& u+v \cdot h_{4}=u+v h^{\perp}+|v| d^{\perp} e^{i \theta_{4}} \\
\Rightarrow & v\left(h_{4}-h^{\perp}\right)=|v| d^{\perp} e^{i \theta_{4}} \\
\Rightarrow & \left|h_{4}-h^{\perp}\right|=d
\end{aligned}
$$

where we used the fact that $v \neq 0$ due to Lemma 1. Thus, $\mathbf{B}_{v}=\mathbf{B}_{u}^{\perp}$ satisfies (5). The other implication is trivial.

\section{Relating to Interference Alignment}

We now relate our coding scheme to the well known interference alignment technique.

Consider the 3 by 1 complex Compound MISO BC. First, we transform the $(2 \times 1)$ complex MISO channel $\mathbf{h}_{j}$ into a $(4 \times 2)$ real channel matrix $\mathbf{H}_{j}$. The channel matrix has now a specific form but let us assume that it is a standard $(4 \times 2)$ real matrix with arbitrary entries. Interference alignment can be applied as follows. For each channel instance $\mathbf{H}_{j}$, the corresponding "user" computes a linear combination of the two real entries observed, let us denote the corresponding channel by $\mathbf{c}_{j}=\mathbf{v}_{j}^{t} \mathbf{H}_{j}$. The newly defined channels $\mathbf{c}_{j}$ are all real MISO channels and span in general 3 real dimensions. Then, one can ensure that for a specific choice of $\left(\mathbf{v}_{1}, \mathbf{v}_{2}, \mathbf{v}_{3}\right)$ the three resulting channel vectors $\left(\mathbf{c}_{1}, \mathbf{c}_{2}, \mathbf{c}_{3}\right)$ span only a 2 dimensional real space. Hence, the interference can be sent orthogonal to the resulting space and thus, each instance will recover 1 real dimension over 1 complex dimension. The resulting DoF is thus $1 / 2$.

In the scheme we suggest, the optimal choice of $(\beta, \alpha)$ are such that for some $\theta_{j}$ :

$$
\begin{aligned}
U & =X_{u}+\alpha_{j} X_{v}+\left(\alpha-\alpha_{j}\right)\left(X_{v}+e^{2 i \theta_{j}} X_{v}^{*}\right) \\
& =\underbrace{X_{u}+\alpha_{j} X_{v}}_{\text {no DoF loss }}+2\left(\alpha-\alpha_{j}\right) e^{i \theta_{j}} \underbrace{\Re\left(e^{-i \theta_{j}} X_{v}\right)}_{\text {half DoF loss }}
\end{aligned}
$$

The first term of this variable leads to no DoF loss since it compensates perfectly the interfering signal $X_{v}$. The last operand is the real part of a rotation of the interfering variable, and thus, it spans only a real dimension and results in no more than half a DoF loss. The two ideas meet then in leaving one real dimension of the setting interference free.

\section{CONCLUSIONS}

In this work, we investigated a new optimal DoF achieving scheme for some complex $J_{y}$ by $J_{z}$ Compound MISO Broadcast Channels. The idea behind this setting is to allow writing on a "bi-chromatic" paper but where we can filter out one of the colours and deal with the remaining one. The suggested scheme is DoF optimal for the 3 by 1 and a class of the 4 by 1 Compound MISO BC. Though the scheme we suggest recovers the same DoF result as Linear Interference Alignment, it is our believe that the non-linear approach is advantageous in non asymptotic SNR regimes. As such, these results come as a first step to constructing capacity achieving schemes for the complex Compound MISO BC.

\section{APPENDIX A \\ ProOF OF THEOREM 1}

\section{A. Improper Gaussian Random Variables}

It is worth mentioning that the signalling scheme used in the proof of Theorem 1 introduces a RV (namely $U$ ) that is complex but not circular in that its Relation Matrix is nonzero. We give here a brief review of related known properties.

Let $\mathbf{U}$ be an $n$-dimensional non-circular complex Random Vector (Improper Complex Gaussian Vector). Then the second moment of $\mathbf{U}$ is defined by two quantities: [13]

- The usual Covariance Matrix $K_{U}=\mathbb{E}\left(\mathbf{U} \mathbf{U}^{H}\right)$.

- The Relation Matrix $M_{U}=\mathbb{E}\left(\mathbf{U} \mathbf{U}^{t}\right)$.

We can define then the covariance matrix of the $2 n$ dimensional "augmented vector" : $\left[\mathbf{U}, \mathbf{U}^{*}\right]$, that we will note $C_{U}$ and which is given by

$$
C_{U}=\left[\begin{array}{cc}
K_{U} & M_{U} \\
M_{U}^{*} & K_{U}^{*}
\end{array}\right]
$$

The entropy of such a complex vector is then given by [14]:

$$
h(\mathbf{U})=\frac{1}{2} \log _{2}\left((\pi e)^{2 n}\left|C_{U}\right|\right) .
$$

\section{B. Proof of Theorem 1}

Fix a channel index $j \in\left\{1, \ldots, J_{y}\right\}$. It is clear that:

$$
I\left(U ; Y_{j}\right)-I(U ; V)=h(U \mid V)+h\left(Y_{j}\right)-h\left(U, Y_{j}\right) .
$$

Then, substituting in these expressions the next signalling:

$$
\left\{\begin{aligned}
\mathbf{X} & =\mathbf{X}_{u}+\mathbf{X}_{v} \\
\mathbf{X}_{u} & =X_{u} \mathbf{B}_{u} \\
\mathbf{X}_{v} & =X_{v} \mathbf{B}_{v} \\
U & =X_{u}+\alpha X_{v}+\beta X_{v}^{*} \\
V & =X_{v}
\end{aligned}\right.
$$

with the notation $h_{j, u}=\mathbf{h}_{j}^{t} \mathbf{B}_{u}$, it is easy to see that the main calculation is that of the determinant of the matrix given by:

$$
C_{U, Y}=\left[\begin{array}{ll}
K_{U, Y} & M_{U, Y} \\
M_{U, Y}^{*} & K_{U, Y}^{*}
\end{array}\right]
$$

where :

$$
K_{U, Y}=\left[\begin{array}{cc}
P_{u}+\left(|\alpha|^{2}+|\beta|^{2}\right) P_{v} & h_{j, u}^{*} P_{u}+\alpha h_{j, v}^{*} P_{v} \\
h_{j, u} P_{u}+\alpha^{*} h_{j, v} P_{v} & \left|h_{j, u}\right|^{2} P_{u}+\left|h_{j, v}\right|^{2} P_{v}+N
\end{array}\right]
$$

and

$$
M_{U, Y}=\left[\begin{array}{cc}
2 \alpha \beta P_{v} & \beta h_{j, v} P_{v} \\
\beta h_{j, v} P_{v} & 0
\end{array}\right] .
$$

Resorting then to the Schur complement, we write that:

$$
\left|C_{U, Y}\right|=\left|K_{U Y}\right|\left|K_{U Y}^{*}-M_{U, Y}^{*} \times K_{U, Y}^{-1} \times M_{U, Y}\right| .
$$

The remainder of the proof follows by standard algebraic manipulations. 


\section{REFERENCES}

[1] H. Weingarten, Y. Steinberg, and S. Shamai, "The capacity region of the gaussian multiple-input multiple-output broadcast channel," Information Theory, IEEE Transactions on, vol. 52, no. 9, pp. 3936-3964, 2006.

[2] H. Weingarten, T. Liu, S. Shamai, Y. Steinberg, and P. Viswanath, "The capacity region of the degraded multiple-input multiple-output compound broadcast channel," Information Theory, IEEE Transactions on, vol. 55, no. 11, pp. 5011-5023, 2009.

[3] H.-F. Chong and Y.-C. Liang, "The capacity region of a class of twouser degraded compound broadcast channels," in Information Theory Proceedings (ISIT), 2013 IEEE International Symposium on, 2013, pp 932-936.

[4] H. Weingarten, S. Shamai and G. Kramer, "On the compound MIMO broadcast channel," Information Theory and Applications (ITA 2007), UCSD, Palo-Alto, USA., Ed., Jan. 29-Feb. 22007.

[5] M. Maddah-Ali, "On the degrees of freedom of the compound MISO broadcast channels with finite states," in Information Theory Proceedings (ISIT), 2010 IEEE International Symposium on, 2010, pp. 2273-2277.

[6] S. Jafar, Interference Alignment: A New Look at Signal Dimensions in a Communication Network. Now Publishers, 2011. [Online]. Available: http://books.google.fr/books?id=GfwB7ItK4esC

[7] C. Huang, S. Jafar, S. Shamai, and S. Vishwanath, "On degrees of freedom region of mimo networks without channel state information at transmitters," Information Theory, IEEE Trans. on, vol. 58, no. 2, pp. $849-857$, feb. 2012

[8] R. Tandon, S. Jafar, S. Shamai Shitz, and H. Poor, "On the synergistic benefits of alternating csit for the miso broadcast channel," Information Theory, IEEE Transactions on, vol. 59, no. 7, pp. 4106-4128, 2013.

[9] M. H. M. Costa, "Writing on dirty paper (corresp.)," Information Theory IEEE Transactions on, vol. 29, no. 3, pp. 439-441, 1983.

[10] W. Yu, A. Sutivong, D. Julian, T. Cover, and M. Chiang, "Writing on colored paper," in Information Theory, 2001. Proceedings. 2001 IEEE International Symposium on, 2001, pp. 302-.

[11] K. Marton, "A coding theorem for the discrete memoryless broadcas channel," Information Theory, IEEE Transactions on, vol. 25, no. 3, pp. 306-311, 1979.

[12] S. Shafiee and S. Ulukus, "Achievable rates in gaussian MISO channels with secrecy constraints," in Information Theory, 2007. ISIT 2007. IEEE International Symposium on, 2007, pp. 2466-2470.

[13] B. Picinbono, "Second-order complex random vectors and normal distributions," Signal Processing, IEEE Transactions on, vol. 44, no. 10, pp. 2637-2640, 1996.

[14] Y. Zeng, C. Yetis, E. Gunawan, Y. L. Guan, and R. Zhang, "Transmit optimization with improper gaussian signaling for interference channels," Signal Processing, IEEE Transactions on, vol. 61, no. 11, pp. 28992913, 2013. 